Article

\title{
Modeling Asymmetric Interactions in Economy
}

\author{
Mirosław Lachowicz ${ }^{1, *, \dagger}$ and Henryk Leszczyński ${ }^{2, \dagger}$ \\ 1 Institute of Applied Mathematics and Mechanics, Faculty of Mathematics, Informatics and Mechanics, \\ University of Warsaw, ul. Banacha 2, 02-097 Warsaw, Poland \\ 2 Institute of Mathematics, University of Gdańsk, ul. Wita Stwosza 57, 80-308 Gdańsk, Poland; \\ hleszcz@mat.ug.edu.pl \\ * Correspondence: M.Lachowicz@mimuw.edu.pl \\ + The authors contributed equally to this work.
}

Received: 3 March 2020; Accepted: 28 March 2020; Published: 3 April 2020

check for updates

\begin{abstract}
We consider a general nonlinear kinetic type equation that can describe the time evolution of a variable related to an economical state of an individual agent of the system. We assume asymmetric interactions between the agents. We show that in a corresponding limit, it is asymptotically equivalent to a nonlinear inviscid Burgers type equation.
\end{abstract}

Keywords: opinion dynamics; asymmetric interactions; kinetic equations; integro-differential equations; generalized inviscid Burgers equation

\section{Introduction}

In the present paper, we are dealing with a kinetic theory approach to modeling complex systems in the economy. We refer to an individual economic characteristic of an agent of a large system and to the description of its distribution through a probability density. Such an approach is quite general and may be related to various economical attributes. The essential feature of the modeling is the interaction between agents of the system. The description is usually referred to as the mesoscopic (kinetic) scale. Similarly as in Boltzmann's kinetic theory of gases, the behavior is referred to as a test-agent of the system, in contrast to the purely microscopic scale in which all agents of the system are taken into account—cf. reference [1].

The methods of kinetic theory in similar contexts were used by many authors, see, for example, [2-6] and references therein. We note that the general reference is the book [7].

In reference [8], the methods of mesoscopic kinetic equations were applied to model interactions of risky assets in a portfolio. The aim was to forecast the short-term evolution (within one year) of the efficient risk/return frontier for equity risk. The analysis was referred to as a subset of stocks traded in the Milan Stock Exchange, taking into account its 13 principal components. The paper provided an extended discussion of a bibliography on the subject.

Reference [9] referred to the economic growth theory. The processes of creation and propagation of new technologies in the description of industrial economic development were considered. The paper proposes a difference-differential model, being an analog of the Burgers equation. The aim was to approach the problem related to a description of endogenous economic growth or technical progress. This leads back to Schumpeter's [10] idea of division of the mechanism of technological development into two separate strategies of innovation and imitation. It is reasonable to assume a continuous variable stating the relationship between these two extrema. The mathematical approaches referred to as Schumpeterian dynamics were developed in many papers-see [11] and references therein. The approach leads to various Burgers-type equations.

Reference [12] modeled a financial market. It was treated as an open system with money exchange with the outside, and a non-equilibrium model was applied. The model takes into consideration 
interactions, nonlinear dynamics and symmetry breaking effects. Reference [13] dealt with a class of underlying mean-reverting stochastic processes in an intensity based model assuming the equilibrium market. Stock price distribution with stochastic volatility is studied in reference [14]. Reference [15] is concerned with the risk premium on the market portfolio of risky assets. The authors show that the risk premium satisfies the Burgers equation.

References [16,17] studied models of opinion dynamics with two types of responses to social influence: conformity and anticonformity. It refers to a description of polarized debates in society (cf. also references in [16,17]. The interplay can lead to a bi-polarized state of the entire system. The creation of bipolar states is also observed for a class of kinetic models-see [6]. In reference [6], it was shown that starting with a unimodal but close to uniform distribution, in the evolution, the occurrence of bimodal (bipolar) distribution is observed. This interesting observation may have importance in the description of political systems in which the political opinions cause the transition from the domination of one party to the coexistence of two different parties in the society-see reference [6] and references therein.

In the present paper, we consider a general nonlinear kinetic type equation that can describe the time evolution of a variable related to an economical state of an individual agent of the system. We stress the possibility of asymmetric interactions between agents. We show that, in a corresponding limit, it is asymptotically equivalent to a nonlinear inviscid Burgers-type equation.

We consider a parameter $v$, representing an economic state of an agent of some population. We apply a mesoscopic description based on the probability density related to a distribution of the state of one test agent of the system. The function $f(t, v)$ describes the probability density to find an agent at the instant of time $t>0$ with state $v \in \Omega$, where $\Omega$ is a domain in $\mathbb{R}^{d}$.

We consider the following general class of mesoscopic equations:

$$
\frac{\partial}{\partial t} f(t, v)=\int_{\Omega} T_{f}(v, w) f(t, w) \mathrm{d} w-f(t, v) \int_{\Omega} T_{f}(w, v) \mathrm{d} w,
$$

where $T_{f}(v, w)$ is the turning rate of state $w \in \Omega$ to state $v \in \Omega$ caused by interaction with other agents. The modeling process leads to the proper choice of the function $T_{f}$ that may depend on state distribution $f$. Such a form of general equations was proposed in reference [18].

We note that similar general structures were proposed by Othmer et al. in [19] and studied, for example, in [20,21] (see also references therein). A detailed bibliographical review can be found in [22]. Various kinetic equations were discussed in [1]. Reference [4] dealt with the dynamics of human crowds and proposed a computational modeling approach. The social interactions were taken into account, and their influence on the behavioral dynamics was defined in the framework of the kinetic theory of active particles. The method was applied to take into account the individual emotional state in the evacuation of a metro station.

In contrast to the previous attempts [6,18,22-24], in the present paper, we study an asymmetric way of interactions. The symmetric interactions resulting in nonlinear diffusion equations were considered in reference [24].

One may see a relationship between the asymmetric interactions and the situation of asymmetric information, see [25], i.e., when one agent has better information than the other, which can lead to degradation of a market.

Assuming

$$
T_{f}(v, w)=\int_{\Omega} A\left(v, w, w^{\prime}\right) \kappa\left(w, w^{\prime}\right)\left(f\left(t, w^{\prime}\right)\right)^{\gamma} \mathrm{d} w^{\prime},
$$

where $\gamma>0$ describes the strength of influence of other agents on the state of a given test agent. The bigger $\gamma$, the stronger the influence is. The function $\kappa\left(w, w^{\prime}\right)$ denotes the rate of influence on the agents with states $w$ by the agent with state $w^{\prime}$, whereas $A\left(v, w, w^{\prime}\right)$ describes the transition probability 
from state $w$ to state $v$ as a result of interaction with the agent with state $w^{\prime}$. Therefore, it is natural to assume that $A: \Omega^{3} \rightarrow \mathbb{R}_{+}$is a measurable function such that

$$
A \geq 0, \quad \int_{\Omega} A\left(v, w, w^{\prime}\right) \mathrm{d} v=1, \quad \forall w, w^{\prime} \in \Omega .
$$

This leads to the following equation

$$
\begin{aligned}
& \frac{\partial}{\partial t} f(t, v)=\iint_{\Omega} A\left(v, w, w^{\prime}\right) \kappa\left(w, w^{\prime}\right)\left(f\left(t, w^{\prime}\right)\right)^{\gamma} f(t, w) \mathrm{d} w \mathrm{~d} w^{\prime} \\
& -f(t, v) \int_{\Omega} \kappa\left(v, w^{\prime}\right)\left(f\left(t, w^{\prime}\right)\right)^{\gamma} \mathrm{d} w^{\prime} .
\end{aligned}
$$

We may note that Equation (3) can be directly related to the dynamics of $N$ interacting agents in the limit $N \rightarrow \infty$-see [1,5], where the interaction between $\gamma$ agents are allowed, and $\gamma$ is an integer.

We assume that

$$
A\left(v, w, w^{\prime}\right)=\delta\left(v-w^{\prime}\right)
$$

in order to obtain the following mesoscopic equation (cf. [18,22-24])

$$
\frac{\partial}{\partial t} f(t, v)=(f(t, v))^{\gamma} \int_{\Omega} \kappa(w, v) f(t, w) \mathrm{d} w-f(t, v) \int_{\Omega} \kappa(v, w)(f(t, w))^{\gamma} \mathrm{d} w .
$$

The simplest choice could be $\gamma=1$; however, the case $\gamma>1$ leads to more difficult considerations involving possibilities of blow-ups in a finite time, see [22-24]. The previous studies of Equation (4) were concentrated on symmetric interactions, that is the symmetric function $\kappa$. In the present paper, we are dealing with asymmetric interactions; that is, we relax the assumption on the symmetry of $\kappa$.

The paper is organized as follows. In Section 2, the formal limit of the scaled kinetic equation with the asymmetric interactions rate is proposed. Preliminary mathematical results related to the kinetic equation are contained in Section 3. Two different local existence results for the Generalized Inviscid Burgers Equation are proposed in Section 4. The main results of the paper, namely the results on the asymptotic relationships between the kinetic and Burgers equations, are stated in Section 5 . The last section is devoted to concluding remarks.

\section{Formal Limit}

Let $\Omega$ be either $\mathbb{R}^{d}$ or $\mathbb{T}^{d}$, where the latter is the $d$-1-dimensional torus in $\mathbb{R}^{d}$. In the case of $\mathbb{T}^{d}$, we adhere to the convention that all mathematical operations are understood in the sense of $\mathbb{T}^{d}$.

We consider Equation (4) with $\kappa(w, v)=\tilde{\kappa}(w-v)$ (we skip tilde for simplicity) and introduce a small parameter $\varepsilon$ leading to the following scaled equation

$$
\begin{aligned}
\frac{\partial}{\partial t} f(t, v) & =\frac{1}{\varepsilon^{d+v}}\left((f(t, v))^{\gamma} \int_{\Omega} \kappa\left(\frac{w-v}{\varepsilon}\right) f(t, w) \mathrm{d} w\right. \\
& \left.-f(t, v) \int_{\Omega} \kappa\left(\frac{v-w}{\varepsilon}\right)(f(t, w))^{\gamma} \mathrm{d} w\right),
\end{aligned}
$$

where the small parameter $\varepsilon>0$ describes the "shrinking" interaction effect and $v=0$ or $v=1$.

Introducing the new variable

$$
w^{\prime}=\frac{w-v}{\varepsilon}
$$


we observe that the right-hand side $\mathcal{R}_{\mathcal{E}}(f)$ can be written as

$$
\begin{aligned}
\mathcal{R}_{\mathcal{\varepsilon}}(f)(t, v) & =\frac{1}{\varepsilon^{v}}\left((f(t, v))^{\gamma} \int_{\Omega} \kappa\left(w^{\prime}\right) f\left(t, v+\varepsilon w^{\prime}\right) \mathrm{d} w^{\prime}\right. \\
& \left.-f(t, v) \int_{\Omega} \kappa\left(-w^{\prime}\right)\left(f\left(t, v+\varepsilon w^{\prime}\right)\right)^{\gamma} \mathrm{d} w^{\prime}\right),
\end{aligned}
$$

and Equation (5) takes the form

$$
\frac{\partial}{\partial t} f(t, v)=\mathcal{R}_{\varepsilon}(f)(t, v) .
$$

Let $\kappa$ be a nonnegative integrable function such that

$$
\begin{aligned}
& B_{0}=\int_{\Omega} \kappa(w) \mathrm{d} w=\int_{\Omega} \kappa(-w) \mathrm{d} w, \\
& B_{1, i}=\int_{\Omega} \kappa(w) w_{i} \mathrm{~d} w=-\int_{\Omega} \kappa(-w) w_{i} \mathrm{~d} w, \quad i=1, \ldots, d,
\end{aligned}
$$

are bounded.

In such a case, $\mathcal{R}_{\varepsilon}(f)(t, v)$ asymptotically behaves like $\varepsilon^{1-v} \mathcal{R}(f)(t, v)$, where

$$
\begin{aligned}
\mathcal{R}(f)(t, v) & =(f(t, v))^{\gamma} \int_{\Omega} \kappa\left(w^{\prime}\right) \frac{\partial}{\partial v} f(t, v) \cdot w^{\prime} \mathrm{d} w^{\prime} \\
& -f(t, v) \int_{\Omega} \kappa\left(-w^{\prime}\right) \frac{\partial}{\partial v}(f(t, v))^{\gamma} \cdot w^{\prime} \mathrm{d} w^{\prime},
\end{aligned}
$$

where '.' denotes the scalar product in $\mathbb{R}^{d}$.

Therefore, we obtain the following Generalized Inviscid Burgers equation

$$
\frac{\partial}{\partial t} f(t, v)=\varepsilon^{1-v}(\gamma+1)(f(t, v))^{\gamma} B_{1} \cdot \frac{\partial}{\partial v} f(t, v) .
$$

in which, for $v=0$, the parameter $\varepsilon$ is explicitly present. On the other hand, the scaling with $v=1$ in Equation (5) would lead to the version of Equation (9) without the presence of $\varepsilon$. This is an analogous effect to that of hydrodynamic limits-cf. $[1,26]$ and references therein.

Expanding $\mathcal{R}_{\varepsilon}(f)(t, v)$, we obtain

$$
\begin{aligned}
& \mathcal{R}_{\varepsilon}(f)(t, v)= \\
& \varepsilon^{1-v} \sum_{i=1}^{d} B_{1 i}\left((f(t, v))^{\gamma} \frac{\partial f(t, v)}{\partial v_{i}}+f(t, v) \frac{\partial(f(t, v))^{\gamma}}{\partial v_{i}}\right)+ \\
& \frac{\varepsilon^{2-v}}{2 !} \sum_{i, j=1}^{d} B_{2 i j}\left((f(t, v))^{\gamma} \frac{\partial^{2} f(t, v)}{\partial v_{i} \partial v_{j}}-f(t, v) \frac{\partial^{2}(f(t, v))^{\gamma}}{\partial v_{i} \partial v_{j}}\right)+ \\
& \frac{\varepsilon^{3-v}}{3 !} \sum_{i, j, k=1}^{d} B_{3 i j k}\left((f(t, v))^{\gamma} \frac{\partial^{3} f(t, v)}{\partial v_{i} \partial v_{j} \partial v_{k}}+f(t, v) \frac{\partial^{3}(f(t, v))^{\gamma}}{\partial v_{i} \partial v_{j} \partial v_{k}}\right)+
\end{aligned}
$$

where $B_{2 i j}=\int_{\Omega} w_{i}{ }_{i} w^{\prime}{ }_{j} \kappa\left(w^{\prime}\right) \mathrm{d} w^{\prime}$ and $B_{3 i j k}=\int_{\Omega} w_{i}{ }_{i} w^{\prime}{ }_{j} w^{\prime}{ }_{k} \kappa\left(w^{\prime}\right) \mathrm{d} w^{\prime}$. It follows that if $\gamma=1$, the second-order term vanishes (as well as any even-order term). On the other side, if $\gamma>1$, then the second-order term results in an anti-diffusive behavior. This leads to the conclusion that the case of $\gamma>1$ is of a singular nature. As we will see (Remark 1 ), the case $\gamma=1$ can also behave singularly.

\section{Kinetic Equation}

The simplest choice is Equation (5) with $\gamma=1$. In contrast to symmetric $\kappa$, Equation (5) is no more trivial if we do not assume the symmetry of $\kappa$. In this case, we can apply the approach in $L_{1}(\Omega)$ and we may note that this does not hold in case $\gamma>1$. 
We observe that the operator $\mathcal{R}_{\mathcal{\varepsilon}}$ is Lipschitz continuous in $L_{1}(\Omega)$, i.e.,

$$
\left\|\mathcal{R}_{\mathcal{E}}(f)-\mathcal{R}_{\mathcal{\varepsilon}}(g)\right\|_{L_{1}} \leq \text { const. }\left(\|f\|_{L_{1}}+\|g\|_{L_{1}}\right)\|f-g\|_{L_{1}}, \quad \forall f, g \in L_{1},
$$

where $\|\cdot\|_{L_{1}}$ is the norm in $L_{1}=L_{1}(\Omega)$, and moreover satisfies the following conservativity property

$$
\int_{\Omega} \mathcal{R}_{\mathcal{\varepsilon}}(f)(v) \mathrm{d} v=0, \quad \forall f \in L_{1} .
$$

A local (in time) existence-uniqueness result in $L_{1}(\Omega)$ is then obtained from Equation (11). By the form of Equation (7), the solution $f=f(t)$ is a priori nonnegative and, by Equation (12), its $L_{1}(\Omega)$-norm is conserved for initial datum $f(0)=f_{0}$, which is a nonnegative element of $L_{1}(\Omega)$. Therefore, we can extend the solution to any $t>0$ we obtain the following global (in time) result.

Proposition 1. Let $\gamma=1, v=0$ or $1, \varepsilon>0$, and $f_{0}$ be a probability density. Additionally, let $\kappa \in L_{\infty}(\Omega)$. Then, there exists a unique solution $f=f(t)$ of Equation $(7)$ in $L_{1}(\Omega)$. For any $t \in[0, \infty]$, the function $f=f(t)$ is a probability density.

Now we consider an arbitrary $\gamma \geq 1$. Our aim is to show the existence of $t_{0}>0$ independent of $\varepsilon$, such that there exists a (unique) solution to Equation (5). The operator $\mathcal{R}_{\varepsilon}$ is a Lipschitz continuous in $L_{\infty}(\Omega)$, so a local existence-uniqueness result follows. It is clear that in the case $v=0$, the existence-uniqueness result follows a time interval $\left[0, t_{0}\right]$, where $t_{0}$ is independent of $\varepsilon$.

Proposition 2. Let $\gamma \geq 1, v=0$ or $1, \varepsilon>0, \kappa \in L_{1}(\Omega)$ and $f_{0}$ be a probability density, such that $f_{0} \in L_{\infty}(\Omega)$. Then, there exists $T>0$, which is independent of $\varepsilon$, such that, on the interval of time $\left[0, \varepsilon^{v} T\right]$, there exists a unique solution $f=f(t)$ of Equation $(7)$ in $L_{1}(\Omega) \cap L_{\infty}(\Omega)$. For any $t \in\left[0, \varepsilon^{v} T\right]$, the function $f=f(t)$ is a probability density.

Proof. It is obvious that the operator $\mathcal{R}_{\varepsilon}$ on the right-hand side of Equation (7) satisfies the (local) Lipschitz condition in $L_{\infty}(\Omega)$ as well as in $L_{1}(\Omega) \cap L_{\infty}(\Omega)$. We show an a priori estimate of the solution. To this end, we derive the integral inequality

$$
|f(t)|_{L_{\infty}} \leq\left|f_{0}\right|_{L_{\infty}}+2 \varepsilon^{-v} B_{0} \int_{0}^{t}|f(s)|_{L_{\infty}}^{\gamma+1} d s,
$$

where $|\cdot|_{L_{\infty}}$ denotes the norm in $L_{\infty}=L_{\infty}(\Omega)$.

Hence, by the comparison of the ODE theorem, we obtain

$$
|f(t)|_{L_{\infty}} \leq \frac{\left|f_{0}\right|_{L_{\infty}}}{\left(1-C_{0} \varepsilon^{-v} t\right)^{\frac{1}{\gamma}}}
$$

with some constant $C_{0}$ dependent on $\left|f_{0}\right|_{L_{\infty}}$.

For $v=1$, the Lipschitz constant depends on $\varepsilon$, and the same can be true for the time-interval of the existence of the solution.

Continuing with $v=0$, we may write the equations for the derivatives of the solution given by Proposition 2. Keeping in mind Equation (6) with $v=0$, we can study the smoothness of solutions. Let $W^{m, p}(\Omega)$ and $C_{B}^{m}(\Omega)$ be the Banach spaces-the classical Sobolev space (a subspace of $L_{p}(\Omega)$ ) -and the space of $m$-differentiable functions, respectively, with the usual norms denoted by $\|\cdot\|_{p}^{(m)}$ and $\|\cdot\|_{[B]}^{(m)}$, respectively, cf. reference [27]. 
Let $X^{(m)}=W^{m, 1}(\Omega) \cap C_{B}^{m}(\Omega), m=0,1,2, \ldots$, and $\|.\|^{(m)}$ be defined

$$
\|\cdot\|^{(m)}=\|\cdot\|_{1}^{(m)}+\|\cdot\|_{[B]}^{(m)}, \quad m=0,1,2, \ldots .
$$

In particular, for $m=0$, we write $X=X^{(0)}=L_{1}(\Omega) \cap L_{\infty}(\Omega)$ and $\|\cdot\|=\|\cdot\|^{(0)}$.

Proposition 3. Let $\gamma \geq 1, v=0$ or $1, \varepsilon>0, \kappa \in L_{\infty}(\Omega)$ and $f_{0}$ be a probability density, such that $f_{0} \in X^{(m)}$ and $\kappa \in X^{(m)}$ for some $m=1,2,3, \ldots$ Then, there exists $T>0$, such that the solution $f=f(t)$ (given by Proposition 2) satisfies $f(t,.) \in X^{(m)}$ for all $t \in\left[0, \varepsilon^{v} T\right]$. If $v=0$, then

$$
\|f(t, .)\|^{(m)} \leq \text { const. } \quad \forall t \in[0, T] .
$$

Proof. The application of Lipschitz-continuity in the spaces $X^{(m)}$ gives the result.

\section{Burgers Equation}

Let $\Omega=\mathbb{R}^{d}$. The result for $\Omega=\mathbb{T}^{d}$ follows analogously. We consider the following equationa version of the Generalized Inviscid Burgers equation

$$
\frac{\partial}{\partial t} f(t, v)=\varepsilon^{1-v} \sigma(v) \cdot\left((f(t, v))^{\gamma} \frac{\partial}{\partial v} f(t, v)\right) .
$$

where $\sigma=\sigma(v)$ is a function $\Omega \rightarrow \Omega$.

Putting $v=1, \sigma \equiv 1$ and $\gamma=1$, we obtain the classical Inviscid Burgers equation. In the general case, Equation (13) need not be conservative in contrast to Equation (9). In fact, $\sigma$ that is constant leads to a conservative case.

Theorem 1. Let $\sigma$ and $f_{0}$ be non-negative, bounded $C^{1}$ functions on $\Omega$, of which the derivatives are bounded. Then:

1. There exist $T>0$ and a unique solution $f$ to Equation (13) on the interval $\left[0, \frac{T}{\varepsilon^{1-v}}\right]$. The function $f$ is $C^{1}$, bounded and nonnegative.

2. If additionally $\frac{\partial^{2}}{\partial v^{2}} f_{0}^{p}$, for some $p \geq 1$, and $\frac{\partial^{2}}{\partial v^{2}} \sigma$ are bounded and continuous, then $f^{p}$ is $C^{2}$ with bounded derivatives $\frac{\partial^{2}}{\partial v^{2}} f^{p}(t, v)$.

3. If $f_{0} \in L_{1}(\Omega)$, then the solution $f(t,.) \in L_{1}(\Omega)$.

Proof. We consider the case $v=1$. For simplicity of notations, we consider a one-dimensional case $d=1$; however, the proof for any $d \geq 1$ can proceed in the same way.

The characteristics $\eta(t)=\eta(t, v)$ are solutions to the ODE problem with papameter $v$

$$
\frac{d \eta(t)}{d t}=-\sigma(\eta(t))\left(f_{0}(v)\right)^{\gamma}, \quad \eta(0)=v
$$

Since $f_{0}$ is bounded and $\sigma$ is Lipschitz continuous, the characteristics $\eta=\eta(t, v)$ exist globally. The solution to Equation (13) is given by the implicit formula

$$
f(t, \eta(t, v))=f_{0}(v),
$$

which is well defined as long as $v \mapsto \eta(t, v)$ is a diffeomorphism. The characteristics do not intersect each other provided that

$$
\frac{\partial}{\partial v} \eta(t, v) \neq 0
$$


Let $\eta_{v}=\frac{\partial}{\partial v} \eta(t, v)$. We consider the corresponding ODE problem

$$
\frac{\mathrm{d} \eta_{v}(t)}{\mathrm{d} t}=-\frac{\mathrm{d}}{\mathrm{d} v} \sigma(\eta(t)) \eta_{v}(t)\left(f_{0}(v)\right)^{\gamma}-\gamma \sigma(\eta(t))\left(f_{0}(v)\right)^{\gamma-1} \frac{\partial}{\partial v} f_{0}(v), \quad \eta_{v}(0)=1
$$

The solution can be estimated as follows

$$
\eta_{v}(t) \geq 1-\int_{0}^{t}\left\{\bar{M}_{\sigma}\left|f_{0}\right|_{L^{\infty}}^{\gamma} \eta_{v}(s)+M_{\sigma}\left|f_{0}\right|_{L^{\infty}}^{\gamma-1}\left|\frac{\partial}{\partial v} f_{0}\right|_{L^{\infty}}^{\gamma}\right\} \mathrm{d} s
$$

where $\bar{M}_{\sigma}=\sup _{v \in \Omega}\left|\frac{\mathrm{d}}{\mathrm{d} v} \sigma(v)\right|$ and $M_{\sigma}=\sup _{v \in \Omega}|\sigma(v)|$.

It is easy to see that $\eta_{v}(t)>0$ on some interval $[0, T]$.

The derivative $\frac{\partial}{\partial v} f(t, v)$ fulfills the formula

$$
\frac{\partial}{\partial v} f(t, \eta(t, v)) \frac{\partial}{\partial v} \eta(t, v)=\frac{\partial}{\partial v} f_{0}(v)
$$

which leads to its boundedness for $t \in[0, T]$.

We consider $p=1$ and observe that the proof for $p>0$ is analogous. The derivatives $\frac{\partial^{2}}{\partial v^{2}} f(t, v)$ fulfill the implicit formula

$$
\frac{\partial^{2}}{\partial v^{2}} f(t, \eta(t, v))\left(\eta_{v}(t, v)\right)^{2}+\frac{\partial}{\partial v} f(t, \eta(t, v)) \frac{\partial^{2}}{\partial v^{2}} \eta(t, v)=\frac{\partial^{2}}{\partial v^{2}} f_{0}(v) .
$$

If $f_{0} \in L_{1}(\Omega)$, then

$$
\int_{\Omega} f(t, \eta(t, v)) \mathrm{d} v=\int_{\Omega} f(t, \eta(t, v)) \eta_{v}(t, v) \frac{1}{\eta_{v}(t, v)} \mathrm{d} v=\int_{\Omega} f_{0}(v) \mathrm{d} v .
$$

Changing the variables $v \mapsto w^{\prime}=\eta(t, v)$, we obtain

$$
\frac{1}{\bar{M}_{\eta}} \int_{\Omega} f\left(t, w^{\prime}\right) \mathrm{d} w^{\prime} \leq \int_{\Omega} f_{0}(v) \mathrm{d} v<\infty,
$$

because $0<\eta_{v}(t, v) \leq \bar{M}_{\eta}$ for $t \in[0, T]$.

We consider an illustrative example.

Example 1. Let $\gamma \geq 1, d=v=1$ and $\kappa(v)=\chi(0 \leq v \leq 1)$, where $\chi($ true $)=1$ and $\chi($ false $)=0$. Equation (5) reads

$$
\frac{\partial}{\partial t} f(t, v)=\frac{1}{\varepsilon^{2}}\left((f(t, v))^{\gamma} \int_{v}^{v+\varepsilon} f(t, w) \mathrm{d} w-f(t, v) \int_{v-\varepsilon}^{v}(f(t, w))^{\gamma} \mathrm{d} w\right)
$$

The corresponding Equation (9) has the form

$$
\frac{\partial}{\partial t} f(t, v)=\frac{\gamma+1}{2}(f(t, v))^{\gamma} \frac{\partial}{\partial v} f(t, v) .
$$

The characteristics are defined

$$
\eta(t, v)=v-t \frac{\gamma+1}{2}\left(f_{0}(v)\right)^{\gamma}
$$


The solution is obtained by

$$
f\left(t, v-t \frac{\gamma+1}{2}\left(f_{0}(v)\right)^{\gamma}\right)=f_{0}(v)
$$

or equivalently

$$
f(t, v)=f_{0}(V(t, v))
$$

where $V=V(t, v)$ is a solution to the nonlinear equation

$$
v=V-t \frac{\gamma+1}{2}\left(f_{0}(V)\right)^{\gamma}
$$

It can be solved on $[0, T]$, such that

$$
0<T<\frac{2}{(\gamma+1) \gamma} \frac{1}{\left|f_{0}\right|_{L^{\infty}}^{\gamma-1}\left|\frac{\partial}{\partial v} f_{0}\right|_{L^{\infty}}}
$$

In particular, for $\gamma=1$, we have

$$
0<T<\frac{1}{\left|\frac{\partial}{\partial v} f_{0}\right|_{L^{\infty}}}
$$

We will provide the analysis involving the Fourier transform.

Now, let $\hat{f}=\mathcal{F} f$ denote the Fourier transform of $f \in \mathcal{S}^{\prime}(\Omega)$, cf. [27], with respect to the variable v,

$$
\hat{f}(k)=\mathcal{F} f(k)=\frac{1}{(2 \pi)^{\frac{d}{2}}} \int_{\Omega} e^{-i k \cdot v} f(v) \mathrm{d} v, \quad k \in \mathbb{R}^{d}, \quad i=\sqrt{-1} .
$$

Let $\mathbb{X}_{p, q}$ denote the completion of the space, which consists of these functions $f$ from $\mathcal{S}^{\prime}(\Omega)$ of which the norm

$$
\|f\|_{p, q}=\sup _{k \in \Omega}\left((1+|k|)^{q} \exp (p(1+|k|))|\hat{f}(k)|\right)
$$

is finite, where $p>0$ and $q>d$, cf. reference [28].

Then, let

$$
\mathbb{X}_{p, q}^{(r)}(I)=\left\{f=f(t): \tilde{f}_{r} \in C\left(I ; \mathbb{X}_{p, q}\right), \tilde{f}_{r}(t, v) \equiv \mathcal{F}^{-1} \exp (-r t|k|) \hat{f}(t, k)\right\}
$$

be the space equipped with the norm

$$
\|f\|_{p, q, I}^{(r)}=\sup _{t \in I}\|f(t)\|_{p-r t, q}
$$

for $r \geq 0$ and a compact interval $I \subset \mathbb{R}^{1}$, where $C(I ; \mathbb{X})$ denotes the space of $\mathbb{X}$-valued continuous functions on $I$.

The following elementary inequalities (with straightforward proofs) are needed throughout the paper

Lemma 1. We have

- If $q_{1}>d$ and $q_{2}>d$, then

$$
\int_{\Omega} \frac{1}{(1+|k-\xi|)^{q_{1}}(1+|\xi|)^{q_{2}}} \mathrm{~d} \xi \leq \frac{\text { const }}{(1+|k|)^{q_{3}}}, \quad \forall k \in \Omega,
$$


where $q_{3}=\min \left\{q_{1}, q_{2}\right\}$.

- If $p>0, r>0$ and $t<\frac{p}{r}$, then

$$
\int_{0}^{t} \exp (-(p-r s)(1+|k|)) \mathrm{d} s \leq \frac{\exp (-(p-r t)(1+|k|))}{r(1+|k|)},
$$

for all $k \in \Omega$.

Lemma 2. Given $p>0, q>d$ and $\delta>0$. Let $\sigma \in \mathbb{X}_{p, q}$. Then, there exists $r>0$ and an interval $I \subset[0, \infty)$, such that $0 \in I$ and

$$
\left\|\varepsilon^{1-v} \int_{I} \sigma(v) \cdot\left(g \frac{\partial}{\partial v} f\right)\right\|_{p, q, I}^{(r)} \leq \delta\|g\|_{p, q, I}^{(r)}\|f\|_{p, q, I}^{(r)}
$$

for any $\varepsilon \in[0,1]$ and $v=0$ or $v=1$.

Proof. Let $p>0, q>d$ and $\delta>0$ be given. It is easy to see that the space $\mathbb{X}_{p, q}^{(r)}(I)$ is an algebra for any $r>0$ and any interval $I$, i.e., if both $g$ and $f$ are in $\mathbb{X}_{p, q}^{(r)}(I)$, then $g f \in \mathbb{X}_{p, q}^{(r)}(I)$. By Lemma 1, we may choose first $r>0$ sufficiently large and then $I$ sufficiently small in order to satisfy Equation (16).

Therefore, we obtain the existence-uniqueness result for Equation (13) on a small time interval (independent of $\varepsilon$ ) for both $v=0$ and $v=1$.

Proposition 4. Given $\gamma \geq 1, p>0, q>d, \delta>0$, and $\sigma \in \mathbb{X}_{p, q}$. Let $f_{0}$ be such that $f_{0} \in \mathbb{X}_{p, q}$. Then, there exists $r>0, T>0$ and a unique solution $f$ to Equation (13) in $\mathbb{X}_{p, q}^{(r)}([0, T])$.

\section{Asymptotic Equivalence}

We assume now that conditions guaranteeing both the local existence-uniqueness of solution $f^{\varepsilon}=f^{\varepsilon}(t)$ of Equation (7) and local existence-uniqueness of solution $f_{B}=f_{B}(t)$ of Equation (9), both with $v=0$ or $v=1$, are satisfied. We say that these solutions are asymptotically equivalent on $[0, T]$, $T>0$, if

$$
\sup _{t \in[0, T]}\left|f^{\varepsilon}(t)-f_{B}(t)\right|_{L_{\infty}} \leq \text { const. } \varepsilon^{2-v} .
$$

Theorem 2. Let $\gamma \geq 1, v=0$ and $f_{0}$ be a probability density on $\Omega$, such that $f_{0} \in X^{(2)}$. Moreover, let $\kappa \in X^{(2)}$ satisfy Equation (8) and

$$
\int_{\Omega} w^{2} \kappa(w) \mathrm{d} w \leq \text { const. }
$$

Let $f^{(\varepsilon)}=f^{(\varepsilon)}(t)$, defined on $\left[0, T_{K}\right]$ be a solution of Equation (7) with the initial datum $f_{0}$. If $f_{B}=f_{B}(t)$, defined on $\left[0, T_{B}\right]$, in $X^{(1)}$ is a solution to Equation (9) with the same initial datum $f_{0}$, then $f^{\varepsilon}$ and $f_{B}$ are asymptotically equivalent, i.e.,

$$
\sup _{t \in[0, T]}\left|f^{(\varepsilon)}(t)-f_{B}(t)\right|_{L_{\infty}} \leq \text { const. } \varepsilon^{2}
$$

where $T=\min \left\{T_{K}, T_{B}\right\}$. 
Proof. Let $f^{(\varepsilon)}=f^{(\varepsilon)}(t)$ and $f_{B}=f_{B}(t)$ be the solutions to Equations (7) and (9), respectively, both given on $[0, T]$, where $T=\min \left\{T_{K}, T_{B}\right\}$. For simplicity, we consider $\gamma \in\{1,2,3, \ldots\}$. We have

$$
\begin{aligned}
& \frac{\partial}{\partial t}\left(f^{(\varepsilon)}(t, v)-f_{B}(t, v)\right)= \\
& \left(f^{(\varepsilon)}(t, v)\right)^{\gamma} \int_{\Omega} \kappa\left(w^{\prime}\right)\left(f^{(\varepsilon)}\left(t, v+\varepsilon w^{\prime}\right)-f^{(\varepsilon)}(t, v)\right) \mathrm{d} w^{\prime} \\
& -f^{(\varepsilon)}(t, v) \int_{\Omega} \kappa\left(w^{\prime}\right)\left(\left(f^{(\varepsilon)}\left(t, v-\varepsilon w^{\prime}\right)\right)^{\gamma}-\left(f^{(\varepsilon)}(t, v)\right)^{\gamma}\right) \mathrm{d} w^{\prime} \\
& -\varepsilon(1+\gamma)\left(f_{B}(t, v)\right)^{\gamma} B_{1} \cdot \frac{\partial}{\partial v} f_{B}(t, v),
\end{aligned}
$$

We use Taylor's expansion and obtain

$$
\begin{aligned}
& \frac{\partial}{\partial t}\left(f^{(\varepsilon)}(t, v)-f_{B}(t, v)\right)= \\
& \varepsilon(1+\gamma)\left(f_{B}(t, v)\right)^{\gamma} B_{1} \cdot \frac{\partial}{\partial v}\left(f^{(\varepsilon)}(t, v)-f_{B}(t, v)\right)+ \\
& \varepsilon(1+\gamma)\left(\left(f^{(\varepsilon)}(t, v)\right)^{\gamma}-\left(f_{B}(t, v)\right)^{\gamma}\right) B_{1} \cdot \frac{\partial}{\partial v} f^{(\varepsilon)}(t, v)+ \\
& \frac{\varepsilon^{2}}{2}\left(f^{(\varepsilon)}(t, v)\right)^{\gamma} \sum_{i, j=1}^{d} \int_{\Omega} \kappa\left(w^{\prime}\right) w_{i}^{\prime} w^{\prime}{ }_{j} \frac{\partial^{2}}{\partial v_{i} \partial v_{j}} f^{(\varepsilon)}\left(t, v+\theta_{1}\left(\varepsilon w^{\prime}-v\right)\right) \mathrm{d} w^{\prime} \\
& -\frac{\varepsilon^{2}}{2} f^{(\varepsilon)}(t, v) \sum_{i, j=1}^{d} \int_{\Omega} \kappa\left(w^{\prime}\right) w^{\prime}{ }_{i} w^{\prime}{ }_{j} \frac{\partial^{2}}{\partial v_{i} \partial v_{j}}\left(f^{(\varepsilon)}\left(t, v-\theta_{2}\left(\varepsilon w^{\prime}+v\right)\right)\right)^{\gamma} \mathrm{d} w^{\prime},
\end{aligned}
$$

where $\theta_{1}, \theta_{2} \in[0,1]$ are some intermediate points. We consider the difference $f^{(\varepsilon)}(t, v)-f_{B}(t, v)$ along the characteristics defined in Section 4

$$
\begin{aligned}
& \frac{\partial}{\partial t}\left(f^{(\varepsilon)}(t, v)-f_{B}(t, v)\right)^{\#}= \\
& \varepsilon(1+\gamma)\left(\left(f^{(\varepsilon)}(t, v)\right)^{\gamma}-\left(f_{B}(t, v)\right)^{\gamma}\right)^{\#}\left(B_{1} \cdot \frac{\partial}{\partial v} f^{(\varepsilon)}(t, v)\right)^{\#}+ \\
& \left(\frac{\varepsilon^{2}}{2}\left(f^{(\varepsilon)}(t, v)\right)^{\gamma} \sum_{i, j=1}^{d} \int_{\Omega} \kappa\left(w^{\prime}\right) w^{\prime}{ }_{i} w^{\prime}{ }_{j} \frac{\partial^{2}}{\partial v_{i} \partial v_{j}} f^{(\varepsilon)}\left(t, v+\theta_{1}\left(\varepsilon w^{\prime}-v\right)\right) \mathrm{d} w^{\prime}\right. \\
& \left.-\frac{\varepsilon^{2}}{2} f^{(\varepsilon)}(t, v) \sum_{i, j=1}^{d} \int_{\Omega} \kappa\left(w^{\prime}\right) w_{i}^{\prime} w^{\prime}{ }_{j} \frac{\partial^{2}}{\partial v_{i} \partial v_{j}}\left(f^{(\varepsilon)}\left(t, v-\theta_{2}\left(\varepsilon w^{\prime}+v\right)\right)\right)^{\gamma} \mathrm{d} w^{\prime}\right)^{\#},
\end{aligned}
$$

where $(f(t, v))^{\#}=f(t, \eta(t, v))$-cf. the proof of Theorem 1.

Therefore, under the assumptions of Theorem 2, by Proposition 3, we obtain the statement.

Thanks to the global existence for $\gamma=1$ in $L_{1}$-see Proposition 1 -we can conjecture a weak asymptotic result on the interval $[0, T]$, which is given by Theorem 1 . In fact, the derivatives of the solution in this case exist at any interval of time, but their norms are not independent of $\varepsilon$. Referring to the test function may allow exploiting the estimations in $L_{1}$ in a one-dimensional case. One can use the Taylor expansion, write the equation along characteristics and note the second order terms vanish. This gives a basis for

Conjecture 1. Let $d=\gamma=v=1$ and $f_{0}$ be a probability density on $\Omega$, such that $f_{0} \in X^{(3)}$. Moreover, let $\kappa \in X^{(3)}$ satisfy Equation (8) and

$$
\int_{\Omega} \kappa(w)|w|^{3} \mathrm{~d} w \leq \text { const. }
$$


Let $f^{(\varepsilon)}=f^{(\varepsilon)}(t)$, defined on $[0, T]$ be a solution of Equation (7) with the initial datum $f_{0}$, and let $f_{B}=f_{B}(t)$, defined on $[0, T]$, in $X^{(1)}$ be a solution to Equation (9) with the same initial datum $f_{0}$, where $T>0$ is given by Theorem 1. Then, $f^{\varepsilon}$ and $f_{B}$ satisfy

$$
\sup _{t \in[0, T]}\left|\int_{\Omega} \varphi(v)\left(f^{(\varepsilon)}(t, v)-f_{B}(t, v)\right) \mathrm{d} v\right| \leq \text { const. } \varepsilon,
$$

for each $\varphi \in C^{(3)}(\Omega)$, where the constant "const." may depend on $\varphi$, but it does not depend on $\varepsilon$.

We turn back now to the Fourier analysis in Section 4. Let $v=1$ and $\Delta(t, v)=f^{(\varepsilon)}(t, v)-f_{B}(t, v)$, where, for simplicity of notations, we do not indicate the $\varepsilon$-dependence of $\Delta$. We have

$$
\begin{aligned}
& \frac{\partial}{\partial t} \Delta(t, v)= \\
& \frac{1}{\varepsilon^{d+1}}\left(\left(f^{(\varepsilon)}(t, v)\right)^{\gamma} \int_{\Omega} \kappa\left(\frac{w-v}{\varepsilon}\right) f^{(\varepsilon)}(t, w) \mathrm{d} w-f^{(\varepsilon)}(t, v) \int_{\Omega} \kappa\left(\frac{v-w}{\varepsilon}\right)\left(f^{(\varepsilon)}(t, w)\right)^{\gamma} \mathrm{d} w\right) \\
& -2\left(f_{B}(t, v)\right)^{\gamma} B_{1} \cdot \frac{\partial}{\partial v} f_{B}(t, v) .
\end{aligned}
$$

For $\gamma=1$, we obtain

$$
\frac{\partial}{\partial t} \Delta(t, v)=f^{(\varepsilon)}(t, v) L_{\varepsilon} f^{(\varepsilon)}(t, v)-2 f_{B}(t, v) B_{1} \cdot \frac{\partial}{\partial v} f_{B}(t, v),
$$

where

$$
L_{\varepsilon} f(v)=\frac{1}{\varepsilon^{d+1}} \int_{\Omega}\left(\kappa\left(\frac{w-v}{\varepsilon}\right)-\kappa\left(\frac{v-w}{\varepsilon}\right)\right) f(w) \mathrm{d} w=\frac{1}{\varepsilon} \int_{\Omega}\left(\kappa\left(w^{\prime}\right)-\kappa\left(-w^{\prime}\right)\right) f\left(v+\varepsilon w^{\prime}\right) \mathrm{d} w^{\prime} .
$$

We may note that for $\Omega=\mathbb{T}^{d}$, i.e., $\Omega$ is the $d$-dimensional torus, such that $|\Omega|=1$, the function $f_{\text {eq }} \equiv 1$ is an equilibrium solution (not necessarily unique) in the sense that

$$
\mathcal{R}_{\varepsilon}\left(f_{\mathrm{eq}}\right)=0 \quad \forall \varepsilon>0 .
$$

Then, $L_{\varepsilon}$ is the Fréchet derivative of the operator $f \rightarrow \mathcal{R}_{\varepsilon}(f)$ at $f=f_{\text {eq }}=1$, i.e., the linearization of $\mathcal{R}_{\varepsilon}$ around $f_{\text {eq. }}$.

We observe that, if $\kappa \in L_{1}$ and

$$
\int_{\Omega} \exp \left(i \varepsilon k \cdot w^{\prime}\right)\left(\kappa\left(w^{\prime}\right)-\kappa\left(-w^{\prime}\right)\right) \mathrm{d} w^{\prime}=2 i \int_{\Omega} \sin \left(\varepsilon k \cdot w^{\prime}\right) \kappa\left(w^{\prime}\right) \mathrm{d} w^{\prime}=: i \lambda_{k, \varepsilon},
$$

for all $k \in \Omega$ and all $\varepsilon>0$ and

$$
\widehat{L_{\varepsilon} f}(k)=i \frac{\lambda_{k, \varepsilon}}{\varepsilon} \hat{f}(k),
$$

for all $k \in \Omega$ and all $\varepsilon>0$.

Therefore, the Fourier transform of the right-hand-side of Equation (18) reads

$$
\begin{aligned}
& \mathcal{F}\left(f^{(\varepsilon)} L_{\varepsilon} f^{(\varepsilon)}-2 f_{B} B_{1} \cdot \frac{\partial}{\partial v} f_{B}\right)(t, k)= \\
& \frac{i}{(2 \pi)^{\frac{d}{2}}}\left(\frac{1}{\varepsilon} \int_{\Omega} \hat{f}^{(\varepsilon)}(t, k-\xi) \lambda_{\xi, \varepsilon} \hat{f}^{(\varepsilon)}(t, \xi) \mathrm{d} \xi-2 \int_{\Omega} f_{B}(t, k-\xi) B_{1} \cdot \xi f_{B}(t, \xi) \mathrm{d} \xi\right) .
\end{aligned}
$$


Keeping in mind that we want to have $\Delta(0, v)=0$ (for all $v \in \Omega$ ), the equation for $\Delta$ may be written in the following form

$$
\begin{aligned}
& \hat{\Delta}(t, k)=\frac{i}{(2 \pi)^{\frac{d}{2}}}\left(\int _ { 0 } ^ { t } \left(\int _ { \Omega } \left(\left(\frac{\lambda_{\xi, \varepsilon}}{\varepsilon}-2 B_{1} \cdot \xi\right) \hat{f}_{B}(t, k-\xi) \hat{f}_{B}(t, \xi)+\right.\right.\right. \\
& \left.\left.\left.\frac{\lambda_{\xi, \varepsilon}}{\varepsilon}\left(\hat{f}_{B}(s, k-\xi) \hat{\Delta}(s, \xi)+\hat{\Delta}(s, k-\xi) \hat{f}_{B}(s, \xi)+\hat{\Delta}(s, k-\xi) \hat{\Delta}(s, \xi)\right)\right) \mathrm{d} \xi\right) \mathrm{d} s\right) .
\end{aligned}
$$

By the obvious inequalities

$$
|\sin x| \leq|x|, \quad|x-\sin x| \leq 2|x|, \quad \forall x \in \mathbb{R}^{1},
$$

we obtain

$$
\left|\frac{\lambda_{\xi, \varepsilon}}{\varepsilon}\right|=2\left|\int_{\Omega} \frac{\sin (\varepsilon \xi \cdot w)}{\varepsilon} \kappa(w) \mathrm{d} w\right| \leq 2|\xi|\left|B_{1}\right|,
$$

and

$$
\left|\frac{\lambda_{\xi, \varepsilon}}{\varepsilon}-2 B_{1} \cdot \xi\right|=2\left|\int_{\Omega}\left(\frac{\sin (\varepsilon \xi \cdot w)}{\varepsilon}-\xi \cdot w\right) \kappa(w) \mathrm{d} w\right| \leq 4|\xi|\left|B_{1}\right| .
$$

Moreover, the inequality

$$
|x-\sin x| \leq \frac{x^{2}}{2} \quad \forall x \in \mathbb{R}^{1},
$$

yields

$$
\left|\frac{\lambda_{\xi, \varepsilon}}{\varepsilon}-2 B_{1} \cdot \xi\right| \leq \varepsilon|\xi|^{2} \int_{\Omega} w^{2} \kappa(w) \mathrm{d} w .
$$

We state the following local (in time) result

Theorem 3. Given $\gamma \geq 1, p>0, q>d$. Let $\kappa \in \mathbb{X}_{p, q}$ satisfy Equation (8) and $f_{0} \in \mathbb{X}_{p, q}$. Then, there exists $r>0, T>0$ and unique solutions $f^{(\varepsilon)}$ and $f_{B}$ to Equations (7) and (13), respectively, both in $\mathbb{X}_{p, q}^{(r)}([0, T])$ and both with the same initial datum $f_{0}$. Moreover, if

$$
\int_{\Omega} w^{2} \kappa(w) \mathrm{d} w<\infty
$$

then, $f^{\varepsilon}$ and $f_{B}$ are asymptotically equivalent, i.e.,

$$
\left\|f^{(\varepsilon)}-f_{B}\right\|_{p, q-1,[0, T]}^{(r)} \leq \text { const. } \mathcal{E} .
$$

Proof. We may note that the proof in the case of $\gamma>1$ follows in a similar way as in case $\gamma=1$. Under the assumption of Theorem 3, by Proposition 4, we have the existence and uniqueness of the solution to Equation (13). Then, by Equations (23) and (24), we observe, applying similar methods to that in the proof of Lemma 2, that we can choose $r>0$ sufficiently large and then $T$ sufficiently small, such that the operator defined by the right-hand-side of Equation (21) is contractive in $\mathbb{X}_{p, q}^{(r)}([0, T])$. Therefore, a unique solution $\Delta$ exists in $\mathbb{X}_{p, q}^{(r)}([0, T])$. This implies the existence and uniqueness of the solution to Equation (7) in $\mathbb{X}_{p, q}^{(r)}([0, T])$. Then, applying Equation (25), we obtain Equation (26). This finishes the proof. 
Remark 1. Let $\kappa$ be the characteristic function of the interval $[0,1]$. Let $\Omega$ be the 1-dimensional torus. Then, the operator $L_{\varepsilon}$, defined by Equation (19), reads

$$
L_{\varepsilon} f(v)=\frac{1}{\varepsilon^{2}}\left(\int_{v}^{v+\varepsilon} f(w) \mathrm{d} w-\int_{v-\varepsilon}^{v} f(w) \mathrm{d} w\right)
$$

The unique solution of

$$
\frac{\partial}{\partial t} f=L_{\varepsilon} f
$$

with the initial datum

$$
f(0, v)=\varepsilon \sin \frac{\pi v}{\varepsilon}
$$

is

$$
f(t, v)=\varepsilon \sin \frac{\pi v}{\varepsilon} \cosh \frac{4 t}{\pi \varepsilon}-\varepsilon \cos \frac{\pi v}{\varepsilon} \sinh \frac{4 t}{\pi \varepsilon} .
$$

Therefore, $|f|$ may be arbitrarily large, despite that the initial datum $f(0, v)$ tends to zero as $\varepsilon \rightarrow 0$.

\section{Conclusions}

The asymmetric interaction plays an essential role in the description of phenomena in Mathematical Economy. In fact, usually, the influence of one agent on another is not a vice-versa situation. This leads to complicated mathematical structures-kinetic-type equations with asymmetric kernels. The mathematical theory of such structures is much more complex than the corresponding theory for equations with symmetric kernels-cf. [6,18,22-24]. In fact, at the formal level, we showed the possible singular behavior of the solutions. The present paper is a first step in the description of such mathematical phenomena and, as we believe, will lead to further interesting research. The main open problem is related to Conjecture 1 . We believe that the mathematical theory may provide a better description of economic phenomena.

Author Contributions: Conceptualization, M.L. and H.L.; methodology, M.L. and H.L.; formal analysis, M.L. and H.L.; investigation, M.L. and H.L.; writing-original draft preparation, M.L. and H.L.; writing-review and editing, M.L. and H.L. All authors have read and agreed to the published version of the manuscript.

Funding: M. Lachowicz was supported by the National Science Centre, Poland: Grant 2017/25/B/ST1/00051.

Conflicts of Interest: The authors declare no conflict of interest.

\section{References}

1. Banasiak, J.; Lachowicz, M. Methods of Small Parameter in Mathematical Biology; Birkhäuser: Boston, MA, USA, 2014.

2. Marsan, G.A.; Bellomo, N.; Gibelli, L. Stochastic evolutionary differential games toward a system theory of behavioral social dynamics. Math. Model. Methods Appl. Sci. 2016, 26, 1051-1093. [CrossRef]

3. Bellomo, N.; Soler, J. On the mathematical theory of the dynamics of swarms viewed as complex systems. Math. Model. Methods Appl. Sci. 2012, 22, 1140006. [CrossRef]

4. Bellomo, N.; Gibelli, L.; Outada, N. On the interplay between behavioral dynamics and social interaction in human crowds. Kinet. Relat. Model. 2019, 12, 397-409. [CrossRef]

5. Lachowicz, M. Individually-based Markov processes modeling nonlinear systems in mathematical biology. Nonlinear Anal. Real World Appl. 2011, 12, 2396-2407. [CrossRef]

6. Lachowicz, M.; Leszczyński, H.; Topolski, A.K. Self-organization with small range interactions: Equilibria and creation of bipolarity. Appl. Math. Comput. 2019, 343, 156-166. [CrossRef]

7. Pareschi, L.; Toscani, G. Interacting Multiagent Systems: Kinetic Equations and Monte Carlo Methods; Oxford University Press: Oxford, UK, 2013.

8. Dolfin, M.; Leonida, L.; Muzzupappa, E. Forecasting efficient risk/return frontier for equity risk with a KTAP approach-A case study in Milan Stock Exchange. Symmetry 2019, 11, 1055. [CrossRef] 
9. Henkin, G.M.; Polterovich, V.M. A difference-differential analogue of the Burgers equation and some models of economic development. Disc. Contin. Dyn. Syst. 1999, 5, 697-728. [CrossRef]

10. Schumpeter, J.A. Business Cycles. A Theoretical, Historical and Statistical Analysis of the Capitalist Process; McGraw-Hill: New York, NY, USA, 1939.

11. Henkin, G.M. Burgers type equation, Gelfand's problem and Schumpeterian dynamics. J. Fixed Point Theory Appl. 2012, 11, 199-223. [CrossRef]

12. Halperin, I.; Dixon, M. Quantum equilibrium-disequilibrium: Asset price dynamics, symmetry breaking, and defaults as dissipative instantons. Physica A 2019, 537, 122187. [CrossRef]

13. Wu, J.-L.; Yang, W. Pricing CDO tranches in an intensity based model with the mean revision approach. Math. Comput. Model. 2010, 52, 814-825. [CrossRef]

14. Stein, E.M.; Stein, J.C. Stock price distribution with stochastic volatility: An analytical approach. Rev. Financ. Stud. 1991, 4, 727-752. [CrossRef]

15. Hodges, S.; Carverhill, A. Quasi mean reversion in an efficient stock market: The characterization of Economic equilibria which support Black-Scholes Option pricing. Econ. J. 1993, 103, 395-405. [CrossRef]

16. Siedlecki, P.; Szwabiński, J.; Weron, T. The interplay between conformity and anticonformity and its polarizing effect on society. J. Artif. Soc. Soc. Simul. 2016, 19, 9. [CrossRef]

17. Krueger, T.; Szwabiński, J.; Weron, T. Conformity, anticonformity and polarization of opinion: Insights from a mathematical model of opinion dynamics. Entropy 2017, 19, 371. [CrossRef]

18. Parisot, M.; Lachowicz, M. A kinetic model for the formation of swarms with nonlinear interactions. Kinet. Relat. Model. 2016, 9, 131-164. [CrossRef]

19. Othmer, H.G.; Dunbar, S.R.; Alt, W. Models of dispersal in biological systems. J. Math. Biol. 1988, 26, $263-298$. [CrossRef]

20. Geigant, E.; Stoll, M. Bifurcation analysis of an orientational aggregation model. J. Math. Biol. 2003, 46, 537-563. [CrossRef]

21. Kang, K.; Perthame, B.; Stevens, A.; Velázquez, J.J.L. An integro-differential equation model for alignment and orientational aggregation. J. Diff. Eqs. 2009, 246, 1387-1421. [CrossRef]

22. Lachowicz, M.; Leszczyński, H.; Parisot, M. Blow-up and global existence for a kinetic equation of swarm formation. Math. Model. Methods Appl. Sci. 2017, 27, 1153-1175. [CrossRef]

23. Lachowicz, M.; Leszczyński, H.; Parisot, M. A simple kinetic equation of swarm formation: Blow-up and global existence. Appl. Math. Lett. 2016, 57, 104-107. [CrossRef]

24. Lachowicz, M.; Leszczyński, H.; Puźniakowska-Gałuch, E. Diffusive and anti-diffusive behavior for kinetic models of opinion dynamics. Symmetry 2019, 11, 1024. [CrossRef]

25. Akerlof, G. The market for lemons: Quality uncertainty and the market mechanism. Q. J. Econ. 1970, 89, 488-500. [CrossRef]

26. Banasiak, J.; Lachowicz, M. On a macroscopic limit of a kinetic model of alignment. Math. Model. Methods Appl. Sci. 2013, 23, 2647-2670. [CrossRef]

27. Adams, R.A. Sobolev Spaces; Academic Press: New York, NY, USA, 1975.

28. Ukai, S.; Asano, K. The Euler limit and initial layer of the nonlinear Boltzmann equation. Hokkaido Math. J. 1983, 12, 311-332. [CrossRef]

(C) 2020 by the authors. Licensee MDPI, Basel, Switzerland. This article is an open access article distributed under the terms and conditions of the Creative Commons Attribution (CC BY) license (http:/ / creativecommons.org/licenses/by/4.0/). 